\title{
BCWSN: A dynamic load balancing algorithm for decrease congestion cost in wireless sensor network
}

\author{
Arash Rahbari, Arash Ghorbannia Delavar \\ Department of Computer, Payame Noor University, PO BOX 19395-3697, Tehran, Iran.
}

\begin{abstract}
Lifetime network is one of the important needs in wireless sensor networks. Case studies show that network increased lifetime possible by spending costs. The cost of congestion is one of those cases. The cost of back pressuring and blocking the rout with timer is obtained in the difference literature. We will be given a dynamic balancing algorithm by BCWSN to reduce congestion. By integration of certain parameters in this the proposed method we have achieved the objective function. To achieve a dynamic balance in the network, with the help of this function and provides the detection threshold and assign the proper id, we have proposed strategies to choose the receiver and sender nodes. Finally, we could with reducing the processing time in the BCWSN proposed method increase network lifetime as compared to the previous. (C)2016 All rights reserved.
\end{abstract}

Keywords: Wireless Sensor network, BCWSN algorithm, load balancing, congestion cost.

\section{Introduction}

The expansion of the global network in 2000, congestion control is important application in computer networks. Limited buffer and battery in wireless sensor networks, congestion prevent and control mechanism is considered to researchers [5, 9, 12, 16, 22,

The occurred congestion have been identified in network different locations. Such as near the source, in the middle of the network, points close to the sink [20]. Network topology is effective on avoid congestion and control mechanism algorithm. In the topology graph and tree data packets from children node send to parent nodes and increased congestion in the parent [1, 7, 8, 13, 15].

Email addresses: rahbari.a6@gmail.com (Arash Rahbari), a_ghorbannia@pnu.ac.ir (Arash Ghorbannia Delavar) 
The rate of production rates, and send and receive data packets are effective on the existence of congestion at a location [18, 20]. Request packets are also led to occupy buffer and the occurrence of congestion in the network [1, 4, 18, 19]. Redundancy and the data packets rotate, can lead to occurrence of congestion in the network. Congestion can lead to discontinuation of the rout and gaps in the network [14, 17]. The shortest path is at risk for early completion of the resources. Dense path is also exposed congestion and the resources of the area are in danger of closure [2, 3, 5, 6, 21]. So in this paper by the new method proposed and simulation it in Matlab, results are compared with the results of our studies.

\section{Related work}

Lee and Shiu offered topology control, multi-threshold, energy balance to increase the lifetime of the network [15]. Lee and oh presented a competitive process and id management for the distribution of data packets to increase the lifetime of the network [14]. Castano and Rossi presented solution to increase the lifetime of the network by studying in the graph, with a single sink and multi sink and with timestamp optimal [6].

Modupe and Olugbara studied energy consumption in the topology rectangular in four status [16]. Kacimi and Dhaou used many-to-one method and mostly off in order to balance energy consumption [12]. Wang and Qian used a hierarchical approach in order to balance energy consumption [21].

Rezaee and Yaghmaee provided the routing table that according to the temporal sequence was arranged. Request messages sent and received and offered a method for preventing and controlling of congestion in patient safety network [18]. Amadeo and Molinaro were named datas and with send request storm, the nearest node with the requested content, to provide an appropriate response [4].

Sergiou and Vassiliou identified congestion at the three sites and raised causes congestion data packets concurrent send and queue length overflow. They studied reducing load traffic and increase resources to congestion prevent and control mechanism [20].

Akbus and Turgut studied network with heterogeneous nodes. They achieved environmental information by send request and reduced memory consumption and increased packet delivery rate [1].

Banimelhem and khasawneh proposed a grid-based multipath with congestion avoidance routing protocol as an efficient QoS routing protocol that is suited for grided sensor networks. They used second main nodes for Congestion control [5].

Hedayati and Ghorbannia Delavar proposed the algorithm to reduce retransmissions and attempt to save energy by taking smaller positions for to forward data. This proposed scheme results in better energy efficiency than traditional GBR offers [10].

In done studies, send and receive data packets is leading to congestion. In this situation, the network is in a state of great imbalance. Thus network needs to high costs for return to an appropriate balance. Our proposed method, send and receive data packets is based on balance. In this situation, the network is in a state small imbalance. So there are small cost to get back to an appropriate balance.

\section{The proposed method}

Our study compared with GMCAR, so our studied environment is in the grid. Final destination in a corner of the network. Border sided diagonal and horizontal directions is used. Other specifications are the same except for what is described below. In the GMCAR method density is factor for forwarding packet data. In our proposed method, the balance of resources as factors for forwarding 
the data packets. Therefore, the proposed method is to avoid congestion. GMCAR pays the large imbalances cost (The occurrence of congestion until needs to block the rout). Whereas we will pay the cost of small balance (reducing difference of the buffer occupancy levels in the grid in order to escape from the congestion).

In our proposed method, sink start the network by sending a message. Each node after receiving the first message it sends to its neighbors. Each node calculates its average send time and receive time. Node with lower number, is the main node and others are sub-node. The grid node sends the message to the neighboring grids. This continues until the last grid.

Each node has 2 types of Constant and variable id. The variable id determine the role of node. Identifiers are binary. Their length based on the number of nodes in the grid and calculated from Eq. (3.1):

$$
l e n_{\mathrm{id}}=\left\lfloor\log _{2}^{\mathrm{n}}+1\right\rfloor \quad n=\text { number of grid node. }
$$

The zero id be allocated to the main node. Respectively the average interval calculated between the main nodes, id from one to the number of nodes to grid be allocated to the sub node. Fig. 1 shows three steps from id changes. Id with one number change to greater id. Other ids deducted from a unit (Eq. (3.2)).

$$
i d_{\mathrm{new}}= \begin{cases}\max (i d) & i d_{\text {old }}=1 \\ i d_{\text {old }}-1 & i d_{\text {old }}>1\end{cases}
$$



Figure 1: Three steps of id change

In each grid, node with one id is receive of the data packets and the node with bigger id is sending of data packets (Fig. 2).

Fig. 3 shows that each main node only generates and sends and receives priority data packets. The main node send data packets to the main node neighbours. No data packet receive from its sub node. This prevents wasting energy into the grid.

Fig. 3 shows that each sub- node sends priority data packets to the main node grid of downstream neighbors and sends data packets without priority to the sub-node with one id in grid neighbour with one id. In a grid, the main nodes no exchange data packets with sub nodes.

In the studied methods the causes of congestion have mentioned to transmit data packets a common purpose so that the lead to data packets queue overflow. Blocking route and route replaced methods to control congestion has been introduced. This can make reduce load traffic in current path and it increase in the alternative route. In this situation there is no load balancing . Thus are reduced performance routes to each other. In our proposed method, priority data packets send forward by main nodes and without priority data packets send to forward by sub-nodes. Fig. 11 shows that the 

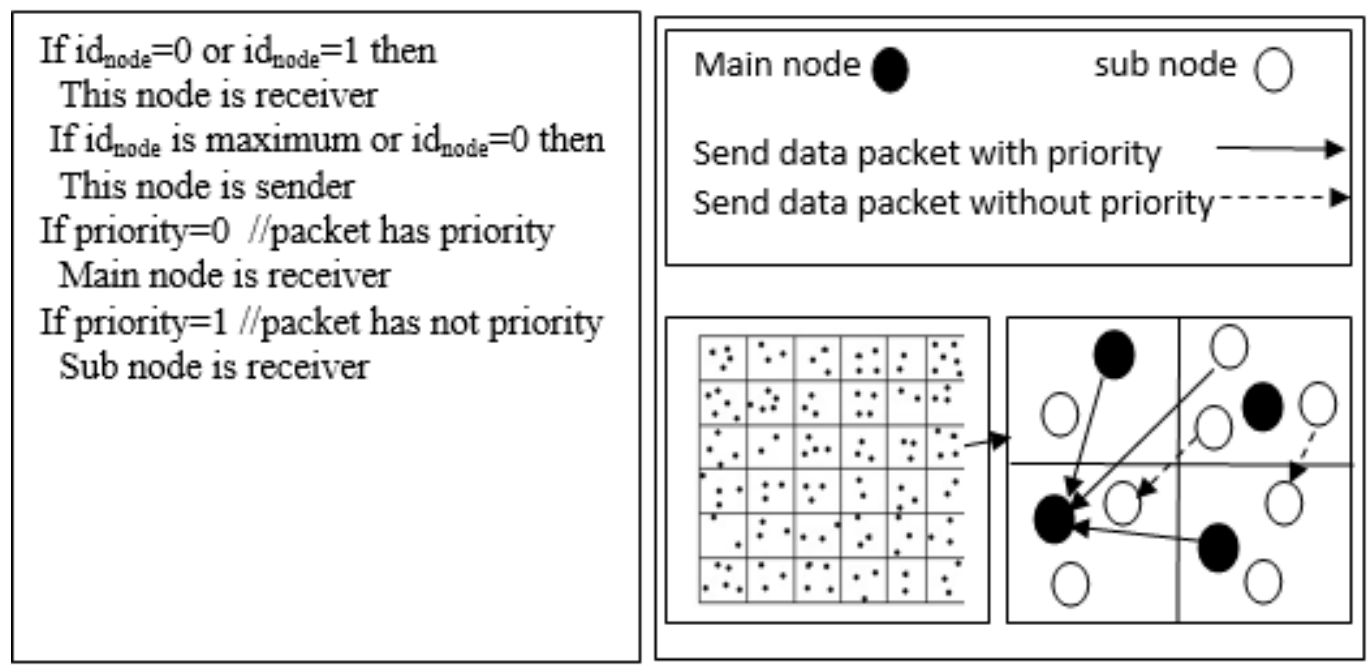

Figure 2: Sender and receiver data packet

role of receiver and sender assigned to all nodes respectively by rotation identifiers. In this way the entry and exit of data packets in the buffer grid nodes is done fairly. In this situation closer balance - the balance of small - were observed in the nodes. When a node is receiver, If does not exist any the data packet for receive or when a node is sender and does not have any data packet to send, gradually, increase the difference in the balance of data packets in the buffer grid nodes increases (Small imbalance).

$/ /$ make the packet with sub node
If id_node $>0$
While view event
$\quad$ Make packet and save packet
End $\{$ if $\}$
End $\{$ when $\}$
$/ /$ send packet in sub node
If packet has priority
Send the packet to selected main
node in to selected neighbor grid
Else
Send the packet to sub node
Figure $3:$

If id_node $=0$
While view event
If information has priority
$\quad$ Make packet and save packet
$\quad$ End $\{$ if $\}$
End $\{$ when $\}$
$/ /$ send packet in main node
If packet has priority
Send the packet to selected main node
in selected neighbor grid
End $\{$ if $\}$


As long as the rotational ID become the same constant ID, ID rotation continues. In this case, each sub node sent to the main node a message containing the number of their buffers data packets. The main node is calculated ID coefficient of variation from Eq. (3.3):

$$
\text { Variance }=s=\sqrt{\frac{1}{n-1} \sum_{i=1}^{n}\left(x_{i}-\bar{X}\right)^{2}} .
$$

$\mathrm{X}_{i}$ is number of data packet in each sub node buffer and $\mathrm{n}$ is number of sub node in this grid and $\mathrm{s}$ is ID coefficient of variation then main node based on Eq. (3.4), determines new sending and receiver nodes and sends new ID for each sub-node. Each sub node stop ID routing after receiving a new ID and id coefficient of variation, until id coefficient of variation become zero.

$$
\operatorname{cost}=\frac{h+t}{b+d}+\lfloor s\rfloor,
$$

$\mathrm{h}$ is hop and $\mathrm{t}$ is time for each hop and $\mathrm{b}$ is level buffer for each node and $\mathrm{d}$ is time of delay for each hop and s is ID coefficient of variation. Node with greater costs number, has lower data packets and is the next receiver data packet. Node with smaller cost number, has more data packets and is sender data packet. Sending and receiving can be by constant sender and receiver nodes. After zero become id coefficient of variation ID routing begins again according Fig. 1 .

ID changes can make reduce the difference in the level of sub-nodes buffer. As the result, it is avoided of the accumulation of data packets (congestion) at a node. As well as data packets discharge from buffered by the higher occupation and of and is entered to buffer with lower occupancy level. Thus plays a role in congestion control.

\section{Performance analysis}

Our proposed method simulation in Matlab environment. Table 1 shows characteristics of the simulated environment. Radio models similar to those in [19] assumed, is used.

$$
\begin{gathered}
E_{T X}=E_{\text {elec }} * k+\epsilon_{\text {amp }} * k * d^{2}, \\
E_{R X}=E_{\text {elec }} * k,
\end{gathered}
$$

$\mathrm{E}_{T X}$ : transfer costs of $\mathrm{K}$ bits message for a distance $\mathrm{d}$ and $\mathrm{E}_{R X}$ : receiver cost of $\mathrm{K}$ bits message and $\mathrm{E}_{\text {elec }}, \epsilon_{\text {amp }}$ are constant.

We show average energy in different parts by Fig. 5. It was observed that our proposed method has saved more energy. Save more energy is because that the balancing of the occupancy level of the buffer alongside power are selection criteria node for the forward packet data. In GMCAR, the node with more energy, selected as the main node, while if its buffer to be full, it will not be a good choice. The node blocked soon and another choice must be made. Node with more energy and empty buffer, is a good choice. Data packets until the block entered into the node, then select the next node in the grid so that there is no balance of resources. In our proposed method the resources changes their situation to balance situation by rotate id and changing the sender and receiver.

In GMCAR not possible the using from blocking route method for congestion avoid and control mechanisms in areas close to the sink. In Our proposed method is possible to send and receive data packets without blocking the route. Rate of delivery of data packets has increased without exchange within Grid and by ID rotate and by reaching balance situation (Fig. 6). 
Table 1: Parameters used in simulation

\begin{tabular}{|l|l|}
\hline Simulation parameter & value \\
\hline Grid size & $50^{*} 50 \mathrm{~m}^{2}$ \\
\hline Number of sensors & 200 \\
\hline Radio range & $142 \mathrm{~m}$ \\
\hline Topology size & $350^{*} 350 \mathrm{~m}^{2}$ \\
\hline Initial sensors energy & $1 \mathrm{~J}$ \\
\hline $\mathrm{E}_{\text {elec }}$ & $50 \mathrm{~nJ} / \mathrm{bit}$ \\
\hline$\epsilon_{\text {amp }}$ & $100 \mathrm{pJ} / \mathrm{bit} / \mathrm{m}^{2}$ \\
\hline Buffer size & $65 \mathrm{data}$ packets \\
\hline
\end{tabular}

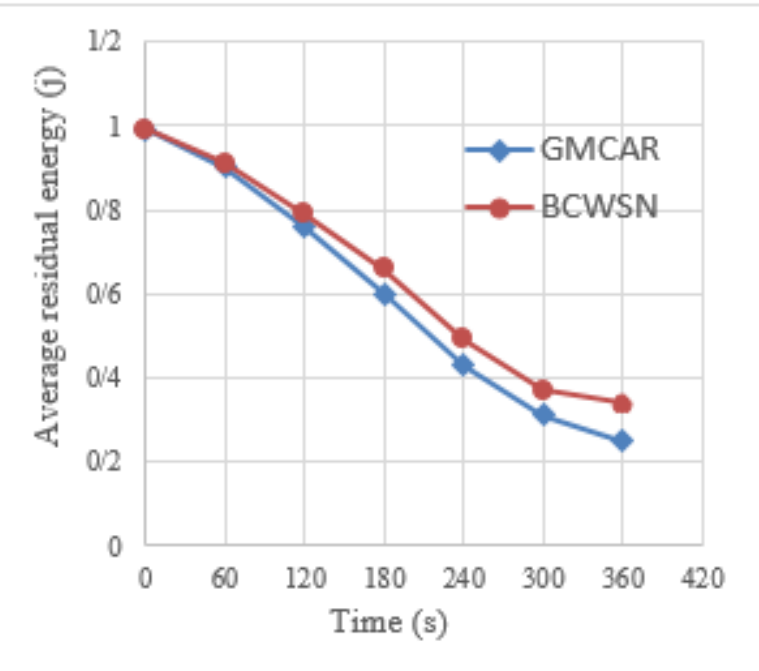

Figure 5: Energy performance.

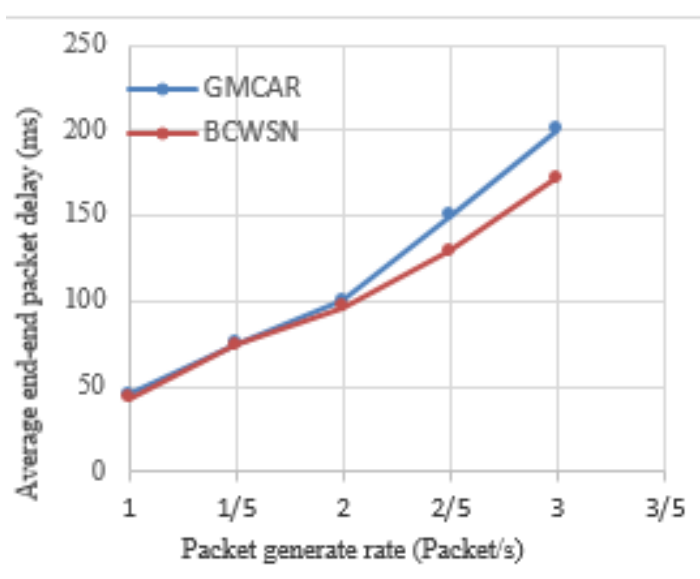

Figure 7: Average end-to-end delay.

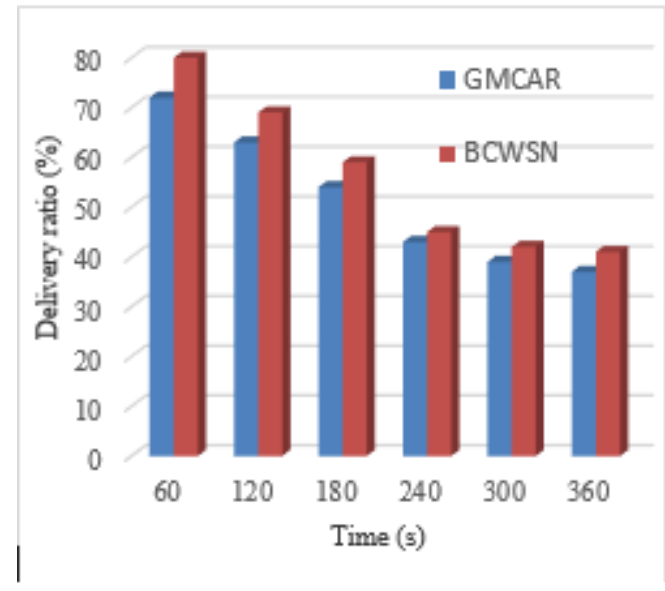

Figure 6: Delivery ratio.

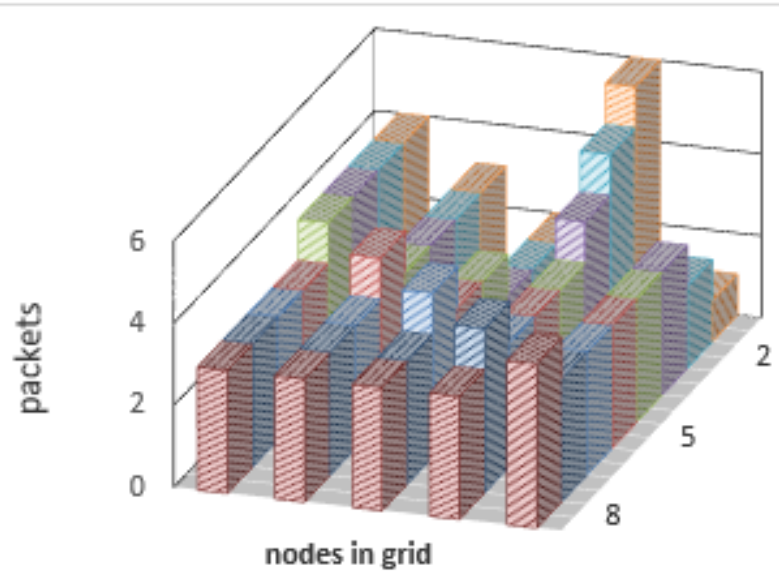

Time (s)

Figure 8: After 8 second, node buffers will be balance.

In GMCAR data packets are guided towards to the high-density areas by less hop. The paths are crowded and reduced performance and the delay increases. In our proposed method changes receiver and sender fairly for establishing balance. Priority packets without are not waiting for priority data 
packets (Fig. 7).

In case increasing imbalances in the buffer, with the proper id change, there will be more balance. Fig. 8 shows an example in a grid with 5 nodes that each node has respectively $(4,3,2,6,1)$ packet in its buffer. After doing our proposed method, their buffers will be balance.

\section{Conclusion}

In studies, different ways to prevent and control congestion has been proposed. By offering this method we reached the conclusion that energy and hop is not enough for determining the next node to exchange data packets and do not provide an appropriate method to prevent and control congestion. Thus, taking into account the level of fair balance in grid nodes alongside the energy and hop increases network lifetime.

\section{References}

[1] M. I. Akbas, D. Turgut, Lightweight routing with dynamic interests in wireless sensor and actor networks, Ad Hoc Networks, 11 (2013), 2313-2328. 1, 2

[2] K. Akkaya, M. Younis, Energy and QoS aware routing for wireless sensor networks, Cluster Comput., 8 (2005), 179-188. 1

[3] J. N. Al-Karaki, A. E. Kamal, Routing techniques in wireless sensor networks: a survey, IEEE Wireless Commun., 11 (2004), 6-28. 1 .

[4] M. Amadeo, A. Molinaro, G. Ruggeri, E-CHANET: Routing,forwarding and transport in InformationCentric multihop wireless networks, Comput. Commun., 36 (2013), 792-803. 1, 2

[5] O. Banimelhem, S. Khasawneh, GMCAR: Grid-based multipath with congestion avoidance routing protocolin wireless sensor networks, Ad Hoc Networks, 10 (2012), 1346-1361. 1, 2

[6] F. Castano, A. Rossi, M. Sevaux, N. Velasco, On the use of multiple sinks to extend the lifetime in connected wireless sensor networks, Electron. Notes Discrete Math., 41 (2013), 77-84. 1, 2

[7] G. H. Ekbatanifard, R. Monsefi, M. H. Yaghmaee, S. A. Hosseini, Queen-MAC: A quorum-based energyefficient medium access control protocol for wireless sensor networks, Comput. Networks, 56 (2012), 2221-2236. 1

[8] M. Eslami, J. Vahidi, M. Askarzadeh, Designing and Implementing a Distributed Genetic Algorithm for Optimizing Work Modes in Wireless Sensor Network, J. math. comput. sci., 11 (2014), 291-299. 1

[9] B. Fateh, M. Govindarasu, Energy minimization by exploiting data redundancy in real-time wireless sensor networks, Ad Hoc Networks, 11 (2013), 1715-1731. 1

[10] S. Hedayati, A. Ghorbannia Delavar, The method of GBR optimization by special parameters to decrease energy consumption in WSNs, J. math. comput. sci., 8 (2014), 387-397. 2

[11] W. R. Heinzelman, A. Chandrakasan, H. Balakrishnam, Energy-efficient communication protocol for wireless sensor networks, IEEE System Sci., 2 (2000), 10 pages.

[12] R. Kacimi, R. Dhaou, A. L. Beylot, Load balancing techniques for lifetime maximizing in wireless sensor networks, Ad Hoc Networks, 11-8 (2013), 2172-2186. 1, 2

[13] J. Kang, Y. Zhang, B. Nath, TARA: Topology-Aware Resource Adaptation to Alleviate Congestion in Sensor Networks, 18 (2007), 919-931. 1

[14] K. S. Lee, S. Oh, C. Kim, A dynamic ID management protocol for CSMA/IC in ad hoc networks, Ad Hoc Networks, 11 (2013), 991-1005. 1, 2

[15] C. Y. Lee, L. C. Shiu, F. T. Lin, C. S. Yang, Distributed topology control algorithm on broadcasting in wireless sensor network, J. Network Comput. Appl., 36 (2013), 1186-1195. 1, 2

[16] I. A. Modupe, O. O. Olugbara, A. Modupe, Minimizing Energy Consumption in Wireless Ad hoc Networks with Meta heuristics, Procedia Comput. Sci., 19 (2013), 106-115. 1, 2

[17] I. H. Peng, Y. W. Chen, Energy consumption bounds analysis and its applications for grid based wireless sensor networks, J. network Comput. Appl., 36 (2013), 444-451. 1

[18] A. A. Rezaee, M. H. Yaghmaee, A. M. Rahmani, A. H. Mohajerzadeh, HOCA: Healthcare Aware Optimized Congestion Avoidance and control protocol for wireless sensor networks, J. Network Comput. Appl., 37 (2014), 216-228. 1, 2 
[19] F. Rouhi, A. Ghorbannia Delavar, S. Hedayati, ETDWSN: A method for energy efficiency increase by combining the index parameters in wireless sensor networks, J. math. comput. sci., 11 (2014), 166-176. 1.

[20] C. Sergiou, V. Vassiliou, A. Paphitis, Hierarchical Tree Alternative Path (HTAP) algorithm for congestion control in wireless sensor networks, Ad Hoc Networks, 11 (2013), 257-272. 1, 2

[21] X. Wang, H. Qian, Research on all-IP communication between wireless sensor networks and IPv6 networks, Comput. Standards \& Interfaces, 35 (2013), 403-414. 1, 2

[22] F. Yan, A. K. H. Yeung, G. Chen, A numerical study of energy consumption and time efficiency of sensor networks with different structural topologies and routing methods, Commun. Nonlinear Science Numer. Simul., 18-9 (2013) 2515-2526. 1 